DOI: https://doi.org/10.46296/yc.v5i8edespab.0094

\title{
LA INTERVENCIÓN SOCIAL Y LA TUTORÍA ESTUDIANTIL COMO MEDIDA DE DISMINUCIÓN DE LOS ÍNDICES DE DESERCIÓN DE LAS UNIVERSIDADES DE LA PROVINCIA DE MANABÍ, PERIODO 2015-2019
}

\section{SOCIAL INTERVENTION AND STUDENT TUTORING AS A MEASURE TO DECREASE THE DROPOUT RATES OF THE UNIVERSITIES OF THE MANABÍ PROVINCE, PERIOD 2015-2019}

\author{
Álvarez-Santana Carmita ${ }^{1 *}$; Caicedo-Montesdeoca Diana ${ }^{2}$ \\ 1 Doctora en Desarrollo Local y Economía Social, docente en Universidad Laica Eloy \\ Alfaro de Manabí (ULEAM). Manta, Ecuador. https://orcid.org/0000-0001-5508-924X \\ ${ }^{2}$ Licenciada en Trabajo Social / Maestrante en Trabajo Social, Mención: Métodos y \\ Técnicas de Investigación. Manta, Ecuador. https://orcid.org/0000-0002-7102-016X
}

*Correo: carmita.alvarez@uleam.edu.ec

\begin{abstract}
Resumen
La educación se ha convertido en una condición fundamental para el desarrollo económico y social de la población, también permite a los individuos aportar elementos indispensables para el ejercicio de sus libertades y capacidades. La deserción universitaria es uno de los fenómenos que afectan a casi todas las instituciones de educación del país y el mundo. Este hecho se manifiesta con la desvinculación del estudiante de sus estudios académicos de manera definitiva o parcial, lo cual es provocado por diferentes factores sean estos económicos, sociales, académicos o personales. A pesar de los crecientes avances que la educación superior a lo largo del tiempo, existen muchos factores que afectan directa o indirectamente a los estudiantes, lo cual podría limitar su permanencia en el proceso de formación, ocasionando la postergación o inclusive abandonó de los estudios. El presente tema de estudio se centraliza en investigar el nivel de intervención profesional de los trabajadores sociales en las Universidades de la Provincia de Manabí y determinar cómo esto influye en la disminución de los índices de deserción universitaria, para lo cual se aplicará una comparación de los índices deserción, así como de la intervención social impartida en los establecimientos. Además, se realizará una proyección cuantitativa de los índices de deserción en los últimos 5 años a nivel general de cada Instituto de educación superior. De esta manera se pretende en base a los resultados proponer alternativas que permitan incrementar la intervención profesional de los trabajadores sociales y en efecto la disminución de los índices de deserción a nivel provincial, fortaleciendo así los indicadores de evaluación del sistema de Educación Superior, y crear alternativas que permitan robustecer a los mismos.
\end{abstract}

Palabras claves: Deserción estudiantil, índices de deserción, intervención social, deserción universitaria.

\begin{abstract}
Education has become a fundamental condition for the economic and social development of the population, it also allows individuals to provide essential elements for the exercise of their freedoms and capacities. University dropout is one of the phenomena that affect almost all educational institutions in the country and the world. This fact manifests itself with the student's disengagement from their academic studies in a definitive or partial way, which is caused by different factors, be they economic, social, academic, or personal. Despite the increasing advances that higher education has made over time, there are many factors that directly or indirectly affect students, which could limit their permanence in the training process, causing the postponement or even abandonment of studies. This study topic focuses on investigating the level of professional intervention of social workers in the Universities of the Province of Manabí
\end{abstract}

Información del manuscrito:

Fecha de recepción: 22 de febrero de 2021.

Fecha de aceptación: 19 de abril de 2021.

Fecha de publicación: 23 de abril de 2021. 
and determining how this influences the decrease in university dropout rates, for which a comparison of the dropout rates, as well as social intervention in the establishments. In addition, a quantitative projection of dropout rates will be carried out in the last 5 years at the general level of each Institute of higher education In this way, based on the results, it is intended to propose alternatives that allow increasing the professional intervention of social workers and, in effect, the reduction of dropout rates at the provincial level, thus strengthening the evaluation indicators of the Higher Education system, and creating alternatives that allow them to be strengthened.

Keywords: Student dropout, dropout rates, social intervention, University dropout.

\section{Introducción}

La Conferencia Mundial sobre Educación para todos de Jomtien (1990), publicada por la Unesco (1990), fijó el objetivo de la educación para todos, dejando enmarcado el acceso libre y voluntario en la educación estudiantil universitaria; mostrando varias líneas de estudio y revisión en curso, lo cual conlleva la creciente atención al fenómeno que se añade directamente a la educación.

Uno de los mayores problemas a los que se enfrenta el mundo hoy día es el aumento mayor de personas excluidas de una participación positiva en la vida educativa, económica, política y cultural. "Una sociedad así no es ni eficaz ni segura". (UNESCO, 2003), creando barreras que impiden bajar el índice de deserción universitaria.

En Ecuador, y en la mayoría de las instituciones educativas de
Latinoamérica, así como lo indica (Barrero Rivera F. , 2015) que "el abandono a la universidades por parte de los estudiantes impacta negativamente el progreso del país en los diferentes campos sociales y científicos" ( $p$ 4), así mismo las causas que ocasionan la deserción estudiantil depende del factor económico, rendimiento académico que es ocasionado como consecuencia de una brecha entre las exigencias de la carrera, y la formación, adquirida en años anteriores a su ingreso a la educación superior.

Según (Machado, 2019, párrafo 1), manifiesta que "El sistema de educación superior ecuatoriano se enfrenta a una paradoja: cada año 133.000 jóvenes que quieren ingresar a la universidades públicas no lo logran, mientras que de los que sí lo lograron, 23.000 optan por desertar del sistema", e indica 
también que a pesar que la oferta educativa en Ecuador está compuesta por 2.037 carreras, el $60 \%$ de los aspirantes sigue optando por 20 carreras tradicionales; lo que produce una deserción, por factores de bajo rendimiento debido a que la carrera no cumplió sus expectativas, el escaso acceso a la información, la falta de orientación, entre otros.

En Manabí en índice de deserción de acuerdo con el informe emitido por la Educación Superior diciembre 2018, en el proceso histórico de oferta de cupos de IES, entre el primer semestre 2012 al primer semestre 2018, se ofertaron inicialmente 92.212 cupos de los cuales el $83 \%$ pudo acceder a un cupo de pregrado. (SENESCYT, 2018, p. 19), por lo tanto, a pesar de que el porcentaje es excelente, en la actualidad mitigar la deserción en educación superior sigue siendo un problema que afecta al mundo.

En la actualidad, el Plan Nacional de Desarrollo de Ecuador indicó que dentro de sus metas 2021 se plantea incrementar del $27,81 \%$ al $31,21 \%$ la tasa bruta de matrícula en educación superior en Universidades y Escuelas Politécnicas al 2021; meta que podría verse limitada por el incremento de los índices de deserción,

El art 174 literal b) de la LOES señala que es función del CACES aprobar la normativa para los procesos de evaluación, acreditación, clasificación académica y aseguramiento de la calidad de las instituciones de educación superior, de las carreras y programas, lo que implica mediante esta evaluación fortalecer el indicador académico que miden a los estudiantes, en el proceso enseñanza, aprendizaje. La tasa de permanencia estudiantil o tasa de retención evalúa el nivel de permanencia e indirectamente el nivel de deserción de los estudiantes de la institución al inicio de su carrera; por lo tanto, los IES debe implementar procesos académicos que garanticen la permanencia de los estudiantes, así como se estable en el Reglamento de Carrera y Escalafón del profesor de Educación Superior (2019), en su Artículo 7, establece las actividades de docencia, entre las cuales menciona dar "Orientación y acompañamiento a través de tutorías presenciales o virtuales, individuales o grupales", permitiendo de esta manera a los docentes dar un acompañamiento 
directo a estudiantes con bajo rendimiento, acompañados de la participación profesional o trabajadores sociales dentro de los IES.

Con esta investigación se promueve conocer como las Tutorías y la Intervención Social juegan un papel importante para bajar los índices de deserción, cuyos resultados obtenidos permite establecer propuestas que mejoren la problemática existente, permitiendo incrementar la intervención profesional de los trabajadores sociales en las instituciones de educación superior, considerando, el factor personal, económico y académico; considerados como los de mayor relevancia, en la deserción universitaria.

\section{Revisión Literaria}

Es preocupante saber la magnitud de deserción estudiantil 0 el abandono en todas las carreras universitarias actualmente, dado que esto conlleva a cuestionar los procesos de carreas y programas que ofrecen las Instituciones de Educación Superior, la Ley Orgánica de Educación Superior (LOES), del año 2018 la cual da un giro sustancial al libre ingreso de las universidades públicas.

(Bazantes, Ruiz Carpio, \& Álvarez Gutiérrez, 2017), manifestó que una de las causas que provoca esta problemática de la deserción de estudiantes universitarios se debe al "bajo nivel de educación, tasas de repetición y deserción escolares elevadas, mala calidad de la educación y deficiente infraestructura educativa y material didáctico son, entre otros, los elementos de mayor incidencia en este tema de la deserción en el Ecuador". (p.65), por lo tanto, esta problemática, es uno de los problemas sociales que influye a la sociedad.

Según la Secretaría de Educación Superior, Ciencia, Tecnología e Innovación (Senescyt), indica que en el 2014 se inscribieron 400.000 estudiantes de pregrado en las universidades y escuelas politécnicas públicas y privadas del Ecuador. De esa cifra, el $26 \%$ abandonó sus estudios.

En el 2015, la Escuela Politécnica Nacional, el rector encargado indicó que "la tasa de deserción fue del 
$50 \%$. El mismo año se inscribieron

5.200 estudiantes en el curso de nivelación de la Politécnica y de esa cantidad, el 33\% aprobó, el 58\% reprobó y el 10\% se retiró. "Una cifra preocupante. Pues detrás de la deserción hay jóvenes frustrados. (Sociedad, 2020, párrafo 7-8), que no ingresaron a la universidad debido al sistema que hay que seguir tener un cupo en sus estudios de este tipo.

Actualmente unas de las alternativas para fortalecer el proceso enseñanza, aprendizaje en las universidades son las tutorías que siguen el acompañamiento al estudiante con el fin de ofrecer la información y las formaciones necesarias para el desarrollo de su carrera y facilitar su inserción en la sociedad como ciudadanos y profesionales activos, lo que "implica un aspecto gradual y acumulativo de experiencias que tienen para cada alumno". (Álvarez Pérez, 2013, p. 8)

Cabe mencionar que las tutorías, así como lo indica (Álvarez Pérez, 2013) son "una tarea integrada en la labor docente, que implica una interacción personalizada del profesor tutor con el alumnado, con la finalidad de hacer un seguimiento del proceso de aprendizaje y definir su proyecto personal de titulación" (p.85).

(Pilar Martínez Clares, Mirian Martínez Juárez, Javier Pérez Cusó, 2016) manifiesta con la idea de disminuir "la tutoría debe aumentar el desarrollo integral del estudiante y favorecer la definición del proyecto profesional y de vida, por lo que se trata de una acción orientadora y formativa que colabora en el desarrollo armónico de los mismos, facilitando la gestión de dicho proyecto, en el cual el tutor es el mediador con relación al mismo". ( $p$. 85)

El Reglamento Institucional de Tutorías Académicas (SITA) de la Universidad Laica Eloy Alfaro de Manabí; sistema que se aplica en las diferentes carreras, indica en el artículo 6 literal b2 que "hay que promover la permanencia de los estudiantes en la carrera que escogieron, mediante la ayuda sistemática y constante, para que el estudiante mejore el rendimiento académico y de esta manera disminuir la deserción estudiantil". (Vicerrectorado Académico, 2016)

En la resolución \#50 del reglamento de funcionamiento de instituciones 
de educación superior, considerando el artículo 26 de la Constitución de la República del Ecuador establece que: "La educación es un derecho de las personas a lo largo de su vida y un deber ineludible e inexcusable del Estado. Constituye un área prioritaria de la política pública y de la inversión estatal, garantía de la igualdad e inclusión social y condición indispensable para el buen vivir. Las personas, las familias y la sociedad tienen el derecho y la responsabilidad de participar en el proceso educativo". (p. 1)

Actualmente en el mundo que vivos y los cambios constantes que sufre el sistema educativo por querer mejorar la calidad de educación creando nuevas estrategias que permita obtener estudiantes de calidad.

Otra problemática empleada que se puede identificar es la deserción universitaria así como lo indica (González Ramírez \& Pedraza Navarro, 2017) es la relación "sociofamiliares que influyen en los estudiantes con riesgo de abandono en las universidades; analizando las relaciones existentes entre abandono universitarios y variables como sexo, edad, nivel educativo y socioeconómico de las familias de origen" (p. 365), provocando el abandono definitivo o transitorio; a la vez que hemos identificado qué factores personales, familiares, institucionales y sociales que son determinantes en la decisión de abandonar una titulación.

(Moncada Mora, 2014), indica que para evitar el abandono de estudiante de la carrera se debe al "nivel de integración que logran los estudiantes" (p.1). Este enfoque se utiliza con el fin de encontrar el factor con la mayor cantidad de información para explicar la decisión de permanecer o abandonar los estudios.

(Moncada Mora, 2014), en la investigación en la zona rural de educación superior indicó que "en el Ecuador la demanda de educación superior en el sistema de estudios a distancia ha tenido un crecimiento regular con una tasa promedio del $10 \%$ semestral; este importante crecimiento se ve fuertemente afectado por la desalentadora tasa de abandono que en promedio afecta al $50 \%$ de cada cohorte de estudiantes". (p. 175) 
El abandono de corto plazo o simplemente el abandono de los estudiantes de nuevo ingreso es uno de los grandes problemas que alteran el comportamiento de los agentes del sistema, es muy conocido que al final del primer ciclo (año o semestre) de estudios es cuando se reduce considerablemente la cohorte de estudiantes.

El trabajador social es un pilar en el proceso de este tipo de problema, cuya finalidad es crear competencias que permitan resolver eficazmente, oportuna el proceso que requiera, crear competencias que se deben adquirir "para la resolución de problemas sociales". (Alcívar Medranda \& Rezabala Farfán, 2017), por lo tanto, la intervención del trabajador social y las unidades competentes serían un pilar fundamental en los procesos de deserción de los estudiantes en las Universidades.

En la actualidad, la deserción y la repitencia constituyen fenómenos que subsisten en las instituciones universitarias de América Latina con diferentes magnitudes e implicaciones tanto en lo individual, colectivo y social.
El problema detectado en la presente investigación se enfoca en los índices de deserción de los estudiantes universitarios, la cual genera un impacto a nivel individual en vista de que la deserción se presenta como un obstáculo para que los jóvenes realicen de manera libre el cumplimiento de sus propósitos de vida; a nivel institucional debido a que las IES deben asegurar la retención de los estudiantes hasta la terminación de sus estudios académicos, parámetro que se encuentra dentro de los criterios de evaluación que realiza el Estado de manera frecuente en todas las instituciones educativas; $y$ a nivel nacional, ya que la deserción universitaria supone una limitación al desarrollo y cumplimiento de objetivos nacionales planteado por los diferentes ministerios $y$ organismos.

Además de múltiples estudios relacionado con la deserción universitaria aplicados en Latinoamérica y a nivel nacional evidencian un incremento progresivo de la tasa de deserción de los estudiantes por la ausencia de tutoría y orientación profesional desde el inicio de sus estudios; 
siendo diversas las causas de deserción es necesario una mayor participación profesional de los trabajadores sociales dentro de LAS IES.

A lo largo de toda carrera universitaria, así como en el ámbito laboral y personal de cada persona; se ha podido evidenciar que muchas son las razones por las que un estudiante universitario opta por el abandono de sus estudios. Estas experiencias permiten percibir que dentro de los establecimientos educativos existe poca intervención por parte de los trabajadores sociales o departamentos que lleven un seguimiento oportuno; creando nuevas estrategias a nivel institucional, lo que permitirá fortalecer los indicadores de evaluación por parte del Conejo de Aseguramiento de la Calidad de le Educación Superior - CACES, a las diferentes Instituciones de Educación Superior de la provincia de Manabí.

Por este motivo es fundamental investigar el nivel de intervención profesional de los trabajadores sociales en las instituciones de educación de la provincia de Manabí y así determinar si dicha intervención contribuye a la disminución de los índices de deserción universitaria; en sus dos variables intervención social y tutorías enmarcadas.

\section{Materiales y Métodos}

Uno de los objetivos de esta investigación es estimar la tasa de deserción de las universidades de la provincia de Manabí, en el período 2015-2019, identificando las causas y las posibles soluciones ante este problema que se presentan en casi todas las universidades del sistema universitario ecuatoriano.

\section{a) Diseño metodológico.}

Para realizar esta investigación y alcanzar los objetivos trazados se realizó un enfoque cuantitativo y de tipo descriptivo que corresponde a un tipo de investigación explicativo, logrando determinar la relación de las variables deserción social y tutorías, en la deserción de estudiantes en Universidades de la provincia de Manabí.

b) Participantes de la investigación.

Los campos de estudio seleccionados fueron las 
Universidades Públicas de la

Provincia de Manabí que dieron un giro sustancial al libre ingreso a estudiantes universitarios, específicamente por la Ley Orgánica de Educación Superior (LOES) que entró en vigor en el año 2010 hasta la actualidad.

\section{c) Área de estudio.}

El universo de estudio estuvo conformado por un número total de 1.408 estudiantes en el periodo 2015 a 2019, perteneciente a Universidades públicas de la provincia Manabí dentro de la zona 4.

\section{d) Técnicas empleadas.}

La información obtenida fue recogida mediante una encuesta online que se encuentra estructurada por tres secciones destinadas para estudiantes en vigencia, alumnos que desertaron y departamentos de bienestar estudiantil que involucren al seguimiento de estudiantes de bajo rendimiento. Esto permitió medir a sus dos variables; la Intervención Social y Tutoría, mecanismos que se emplean para reducir este problema en universidades, creando estrategias para mejorar y robustecer el proceso de enseñanza, aprendizaje, producidas por su entorno social, económico, nivel de conocimiento impide que el estudiante culmine sus estudios.

\section{e) Análisis estadístico.}

Para la tabulación de resultados se utilizó el software SPSS V.25 que consiste el recuento de las respuestas obtenidas de la encuesta online; con la finalidad de generar resultados que se muestran en las tablas, permitiendo establecer propuestas que mejoren la problemática existente, y que permitan incrementar la intervención profesional de los trabajadores sociales en las instituciones de educación superior. Para definir el tamaño de la muestra se seleccionó un total de 1.408 estudiantes y se trabajó con un $90 \%$ de confianza, se utilizó una varianza de un 0,5 y un $5 \%$ de error, lo que define una muestra aleatoria de 302 estudiantes de pregrado.

Los resultados obtenidos demuestran que la cantidad de estudiantes desertores, y de estudiantes que aún se mantienen, 
permite establecer los factores que influyen en la trayectoria de estudios.

\section{Resultados}

La recolección de los datos se realizó a través de un formulario online diseñada en Microsoft Forms de office 365 , el mismo fue diseñado para medir el índice de deserción de estudiante, causados por factor económico, social, y record académico entre otros de las Universidades de la provincia Manabí tomando como alternativas para reducir este índice; las tutorías y la intervención social; frente a la gestión que realiza las universidades.

Se aplicó la encuesta a 302 estudiantes: el $73.00 \% \quad(n=219)$ fueron alumnos que siguen en sus estudios y un 27,00 \% (n=83) corresponde a estudiantes desertores, de acuerdo a los resultados obtenidos entre edades comprendidas de 17 a 20, 20 a 29, 30 a 39 y 40 a 49 años, sin embargo, como resultado si en algún momento los estudiantes dejarían sus estudios académicos; se obtuvo que un $54,66 \%$ en una edad comprendida entre 20 a 29 años, están considerando en desertar la carrera, debido a que la misma no cumplió las expectativas debido a la falta de información o por que la carrera fue asignada de acuerdo al puntaje obtenido en la prueba ser Bachiller. Otra de las causas que ocasionan la deserción es por la oportunidad de trabajo, que en muchos casos indican que por el horario que actualmente demanda la carrera y todas las actividades de vinculación e investigación o prácticas le impiden seguir con sus estudios y un 6,06\% indican que no dejarían sus estudios Tabla 1.

Tabla 1. Frecuencia de deserción de estudiantes.

\begin{tabular}{ccc}
\hline \multirow{2}{*}{ Edad } & \multicolumn{2}{c}{$\begin{array}{c}\text { Deserción de } \\
\text { Estudiantes }\end{array}$} \\
\cline { 2 - 3 } & \multicolumn{1}{c}{ Si } & \multicolumn{1}{c}{ No } \\
\hline 17 a 20 & $35,37 \%$ & $39,39 \%$ \\
20 a 29 & $54,66 \%$ & $54,55 \%$ \\
30 a 39 & $9,97 \%$ & $6,06 \%$ \\
Total & $\mathbf{1 0 0 \%}$ & $\mathbf{1 0 0} \%$ \\
\hline
\end{tabular}

Nota: La tabla muestra el índice de deserción de estudiantes universitarios en las Universidades de Manabí en el periodo 2015 al 2019.

Como factores para determinar la deserción que no solamente afecta al estudiante, sino también a la institución de educación superior; por no cumplir con la visión; notando una mayor prevalencia en el factor 
económico con un $49,3 \%(n=143)$ de estudiante y de desertores que indicaron que la deserción de estudiantes es causa del factor económico, mientras que un 5,0\% $(n=15)$ corresponde a maternidad; factor de menor incidencia, lo que indica que no es impedimento para desertar, así como se muestra en la Tabla 2.

Tabla 2. Frecuencia de factores que provocan la deserción.

\begin{tabular}{lccc}
\hline \multirow{2}{*}{$\begin{array}{l}\text { Factor de } \\
\text { Abandono }\end{array}$} & \multicolumn{2}{c}{$\begin{array}{c}\text { Estudiante - } \\
\text { Desertores }\end{array}$} & $\begin{array}{c}\text { Porcentaje } \\
\text { de casos }\end{array}$ \\
\cline { 2 - 3 } & $\mathbf{N}$ & Porcentaje & $3,8 \%$ \\
\hline Estado civil & 11 & $3,6 \%$ & $21,2 \%$ \\
Salud & 62 & $20,5 \%$ & $15,1 \%$ \\
Trabajo & 44 & $14,5 \%$ & $49,3 \%$ \\
Económico & 143 & $47,5 \%$ & $4,5 \%$ \\
Récord & 13 & $4,3 \%$ & $4,8 \%$ \\
Académico & & & $5,1 \%$ \\
Familia & 14 & $4,6 \%$ & \\
Maternidad & 15 & $5,0 \%$ & \\
Total & $\mathbf{3 0 2}$ & $\mathbf{1 0 0 , 0} \%$ & \\
\hline & & &
\end{tabular}

Nota: La tabla muestra los resultados a partir de las causas que ocasionan la deserción de estudios de pregrado en Universidades de Manabí.

Otro factor determinante para indagar y medir los cambios, que ocasionan desde las percepciones del estudiantado acerca del espacio de tutorías, se encontró que el 20,3\% de los sujetos encuestados expresaron no haber recibido y un $79,7 \%$ indicaron que sí han recibido tutorías en algún momento, teniendo un grado de mayor inclinación hacia la elección de buena 44,1\%, mientras que el $4,4 \%$, indicaron que fueron excelente como respuesta de estudiantes de las Universidades de la provincia de Manabí, Tabla 3.

Tabla 3. Frecuencia de grado de aceptación de Tutorías en la Educación Superior.

\begin{tabular}{lccc}
\hline \multirow{2}{*}{ Resultados } & \multicolumn{2}{c}{ Respuestas } & Porcentaje \\
\cline { 2 - 4 } & $\mathbf{N}$ & Porcentaje & $\begin{array}{c}\text { Po casos } \\
\text { de }\end{array}$ \\
\hline Bueno & 139 & $44,1 \%$ & $46,0 \%$ \\
Muy Bueno & 104 & $33,0 \%$ & $34,4 \%$ \\
Malo & 22 & $7,0 \%$ & $7,3 \%$ \\
Regular & 36 & $11,4 \%$ & $11,9 \%$ \\
Excelente & 14 & $4,4 \%$ & $4,6 \%$ \\
Total & $\mathbf{3 1 5}$ & $\mathbf{1 0 0 , 0} \%$ & \\
\hline
\end{tabular}

Nota: La tabla muestra como las tutorías ayudan a que los estudiantes no deserten de sus estudios universitarios, mejorando el proceso enseñanza aprendizaje en Universidades de Manabí.

Como respuesta al tipo de tutorías que perciben se constató de una manera clara la inclinación del estudiante y desertores hacia dos de las opciones. Afirmando que la finalidad de las tutorías universitarias es de apoyo académico en primer lugar en un $92,5 \%(n=258)$, seguido del apoyo Psicológico en 1,4\% ( $n=4)$; así como también se demuestra que el $33,0 \%$ del total de estudiantes y desertores encuestados afirmaron 
que han tenido apoyo por parte del docente fuera del salón de clase, lo que indica que el proceso enseñanza aprendizaje es un mecanismo que permitirá fortalecer este tipo de programa; y un $23,2 \%$ siempre recibieron este tipo de apoyo, así como lo indica la Tabla 4 y una menor inclinación nunca con un $9,2 \%$.

Tabla 4. Frecuencia de Apoyo de Tutorías en la Educación Superior.

\begin{tabular}{lccc}
\hline Respuestas & $\mathbf{N}$ & Porcentaje & $\begin{array}{c}\text { Porcentaje } \\
\text { de casos }\end{array}$ \\
\hline Siempre & 73 & $23,2 \%$ & $24,2 \%$ \\
$\begin{array}{l}\text { Muchas } \\
\text { veces }\end{array}$ & 74 & $23,5 \%$ & $24,5 \%$ \\
$\begin{array}{l}\text { Algunas } \\
\text { veces }\end{array}$ & 104 & $33,0 \%$ & $34,4 \%$ \\
Casi nunca & 35 & $11,1 \%$ & $11,6 \%$ \\
Nunca & 29 & $9,2 \%$ & $9,6 \%$ \\
Total & $\mathbf{3 1 5}$ & $\mathbf{1 0 0 , 0} \%$ & \\
\hline
\end{tabular}

Nota: La tabla muestra los resultados obtenidos de las tutorías como apoyo en el proceso de educación de los estudiantes de pregrado en las Universidades de Manabí.

Otra variable a medir se ve reflejada

a Intervención Social, como factor predominante para reducir la deserción, son los mecanismos a utilizar para darle un acompañamiento a estudiantes que desierta, lo que determinó que un $90,00 \%$ de los encuestados surgieren darle seguimiento de las tutorías académicas impartidas por el docente especializado y un 58\% indica que con la creación de nuevos sistemas de ayudas de tipo económicas o becas evitaremos la deserción y un $20 \%$ indica que las charlas de motivación y de orientación, podrá evitar que el estudiantes deserte.

\section{Discusión}

Los resultados obtenidos del proceso de investigación revelaron que los factores de riesgo de acuerdo a la muestra de estudio en el periodo 2015 al 2019, se comenzó con el diseño y adaptación de un cuestionario online que permitió determinar la intervención social y las tutorías como variables predominantes de aquellos factores que posiblemente estuvieran relacionados con la deserción universitaria, la cual genera un impacto a nivel individual, en vista de que la deserción se presenta como un obstáculo para que los jóvenes realicen de manera libre el cumplimiento de sus propósitos de vida; a nivel institucional debido a que la IES deben asegurar la retención de los estudiantes hasta la terminación de sus estudios académicos, parámetro que se 
encuentra dentro de los criterios de evaluación que realizan de manera frecuente a todas las instituciones educativas; y a nivel nacional ya que la deserción universitaria supone una limitación al desarrollo y cumplimiento de objetivos, lo que conducen a conocer los tipos de desertores.

Finamente podemos indicar que para reducir la deserción de acuerdo con la investigación realizada se platean fortalecer el sistema educativo que actualmente cuentan las universidades, para mejorar el proceso enseñanza aprendizaje, como una alternativa de mayor incidencia en un 39\% $(n=140)$, seguidos en $24,8 \% \quad(n=89)$ que involucran, ampliar el programa de becas y ayuda económica y un $4,7 \%$ $(n=17)$ que indicaron que mejorar la infraestructura y equipamiento para mejorar el aprendizaje.

Todas estas alternativas ayudarán a mitigar la deserción de estudiantes en Universidades de la provincia de Manabí.

\section{Conclusiones}

Las conclusiones obtenidas del resultado de esta investigación son las siguientes:

Las variables de estudio indican que las tutorías y la Intervención Social, juegan un papel primordial en la deserción universitaria que en muchos casos no existen los mecanismos, herramientas 0 unidades creadas para darle el seguimiento adecuado y crear alternativas para reducir el índice de desertores universitarios que afecta a una sociedad que necesita de profesionales competentes para cumplir con la meta o visión institucional en una empresa que requiera de profesionales eficientes en las asignadas.

Cabe mencionar que el factor económico y el sistema que actualmente implantó el sistema de Educación Ecuatoriano exige a los estudiantes escoger la carrera de acuerdo con el puntaje obtenido, lo que implica que la deserción se vea afectada a pesar de que exista alternativas internas para mitigar este tipo de problema. 
7.

Limitaciones

\section{Recomendaciones}

Es importante indicar que esta investigación a pesar de ser un tema de mayor concurrencia actualmente en el sistema de Educación Superior, se pudo determinar que en muchos casos, a pesar de tener un sistema gratuito en este tipo de universidades públicas, no tienen un mecanismo o proceso fijo que faciliten este tipo de información para poder realizar un estudio profundo de deserción estudiantil; por lo que se recomienda integrar los departamentos ya creados destinados a llevar este tipo de problemas, que en su caso se ven afectados al momento de una evaluación institucional de universidades y escuela politécnicas por parte de la CACES

Por tanto, se deja abierta la posibilidad para seguir trabajando futuras investigaciones en el tema que llevan a completar esta iniciativa, que puede contribuir a comprender el fenómeno de la deserción desde el presente abordaje del estudio.

\section{Bibliografía}

Alcívar Medranda, E. M., \& Rezabala Farfán, v. (2017). Las competencias del trabajador social en unidades educativas del cantón Portoviejo: caso de estudio en la unidad educativa Portoviejo. Caribe a de Ciencias Sociales. Obtenido de

https://www.eumed.net/rev/ca ribe/2017/04/unidad-

educativa-portoviejo.html

Álvarez Pérez, P. R. (2014). La función tutorial del profesorado universitario: Una nueva competencia de la labor docente en el contexto del EEES. Revista Portuguesa de Pedagogía, 47(2), Pag. 85-106.

Barrero Rivera, F. (2015). Investigación en deserción estudiantil universitaria: educación, cultura y significados, 9(2). Educación y desarrollo estudiantil, 86101.

Bazantes, Z., Ruiz Carpio, M., \& Álvarez Gutiérrez, M. L. (2017). Deserción estudiantil universitaria en ecuador y su influencia en la calidad del egresado. Magazine de las ciencias, Vol. 1(Núm. 4), 265. Obtenido de https://revistas.utb.edu.ec/ind ex.php/magazine/article/view/ 183 
Ceaaces. (04 de 2015). laen. Obtenido de Reglamento Funcionamiento Instituciones Educación Superior Ceaaces: https://www.iaen.edu.ec/dow nload/compendio/NORMATIV A\%20EXTERNA/REGLAME NTO\%2OFUNCIONAMIENTO \%20INSTITUCIONES\%20ED UCACION\%20SUPERIOR\% 20CEAACES.pdf

González Ramírez, T., \& Pedraza Navarro, I. (2 Jul-Oct de 2017). Variables sociofamiliares asociadas al abandono de los estudios universitarios. Educatio Siglo XXI, 365-388. doi: https://doi.org/10.6018/j/2986 51

Moncada Mora, L. F. (2014). La integración académica de los estudiantes universitarios como factor determinante del abandono de corto plazo. un análisis en el sistema de educación superior a distancia del ecuador-. RIED. Revista lberoamericana de Educación a distancia, V. 17: 2, 2014, pp 173-196 1. Obtenido de https://www.redalyc.org/pdf/3 314/331431248009.pdf

Pilar Martínez Clares, Mirian Martínez Juárez, Javier Pérez Cusó. (2016). ¿Cómo avanzar en la tutoría universitaria? estrategias ¿cómo avanzar en la tutoría universitaria?
ESTRATEGIAS. REOP, Vol. 27, no2, 2ํ Cuatrimestre, pp. $80-98$.

Plan Nacional de Desarrollo 20172021, T. (s.f.). Secretaría Nacional de Planificación y Desarrollo - Senplades 2017. Obtenido de CONSEJO NACIONAL DE PLANIFICACIÓN-CNP.

Sociedad. (2016). El telégrafo. Obtenido de La deserción universitaria bordea el $40 \%$ : https://www.eltelegrafo.com.e c/noticias/sociedad/4/ladesercion-universitariabordea-el-40

UNESCO. (2003). UNESCO. Obtenido de https://sid.usal.es/idocs/F8/F DO23194/superar_la_exclusi on_mediante_planteamientos .pdf

Vicerrectorado Académico ULEAM. (2016). ULEAM. Obtenido de El Órgano Colegiado Académico Superior: https://drive.google.com/file/d /OByzWXkp991pya2pVNWY wQmZzMU0/view 